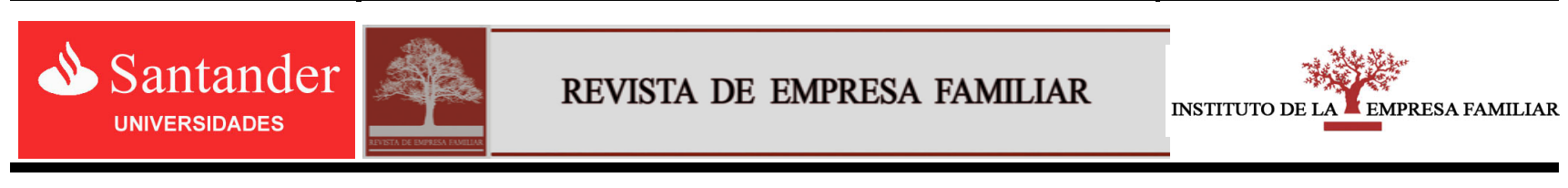

www.revistadeempresafamiliar.uma.es

\title{
Mapa de la cultura organizativa de la empresa familiar: un estudio de casos en la provincia de Córdoba
}

\section{Map of the organizational culture of family firm: a study of cases in the province of Córdoba}

\author{
Maribel Rodríguez Zapatero ${ }^{\text {a,* }}$ \\ a Departamento de Estadística, Econometría, Investigación Operativa y Organización de Empresas. \\ Universidad de Córdoba,14005 (Spain))
}

D A T OS A R T Í C U L O Historial:

Recibido 21-10-2010

Aceptado 29-11-2011

Palabras clave:

Empresa Familiar

Cultura organizativa

Análisis de componentes principales

Códigos JEL:

M19

\begin{tabular}{l} 
A R T I C L E I N F O \\
\hline Article history: \\
Received 21 October 2010 \\
Accepted 29 November 2011
\end{tabular}

Keywords:

Family firm

Organizational culture

Principal components

analysis

JEL codes:

M19
R E S U M E N

En este trabajo se plantea la necesidad de estudiar la cultura organizativa en la empresa familiar como motor de crecimiento, bajo la hipótesis de que la cultura organizativa de la empresa familiar es única e inimitable y su conocimiento y gestión puede ayudar a la supervivencia de la misma. A tal efecto se diseña un cuestionario contestado por 105 individuos de 23 empresas familiares. Se aplica un análisis de componentes principales con el objetivo de obtener un posicionamiento de la empresa familiar en un mapa factorial de la cultura organizativa, pudiendo así detectar qué empresas deben mejorar, en qué deben mejorar, y cuál es la dirección de mejora. Las principales conclusiones halladas son la inexistencia de un patrón repetitivo en la cultura organizacional y la evolución de la misma a lo largo del tiempo.

\section{A B S T R A C T}

In this research, the present work shows the need to consider the organizational culture in the family business as an engine of growth, under the assumption that the organizational culture of the family business is unique and inimitable and its knowledge and management can help the survival of the family firm A questionnaire answered by 105 individuals of 23 family businesses is designed for this purpose. An analysis of principal components is used with the aim of obtaining the family business positioning in a factorial map of organizational culture. It can be detected which companies must improve culture, in what must be improved, and what is the direction of improvement. The main findings found are the absence of a repeating pattern in organizational culture and the evolution of it in time.

\footnotetext{
Autor de contacto. Tel.: +34957218747

Correoselectrónicos: es3rozai@uco.es
} 


\section{Introducción}

No podemos olvidar que estamos trabajando con un ente singular, la empresa familiar, en la que dos sistemas que a la vez son complementarios y antagónicos a la hora de tomar decisiones, ejecutarlas y valorar sus resultados en un mecanismo de retroalimentación, se engranan en busca de la generación de valor. En este trabajo se plantea la necesidad de estudiar la cultura organizativa en la empresa familiar como motor de crecimiento, bajo la hipótesis de que la cultura organizativa de la empresa familiar es única e inimitable y su conocimiento y gestión puede ayudar a la supervivencia de la empresa familiar. Mediante la aplicación de un test encontraríamos la posición de la empresa familiar en la dimensión cultura organizativa, obteniendo una herramienta vital de diagnóstico. En esta situación era importante definir el concepto de cultura o clima organizacional.

\section{Revisión teórica}

El concepto de clima organizacional fue introducido por Gellerman (1960) en el ámbito de la administración de empresas y de la psicología industrial/laboral. Podemos hablar de tres tipos de definiciones sobre el clima organizacional: el primer grupo toma las características meramente objetivas $\mathrm{y}$ estructurales de las empresas (Glick, 1985), un segundo grupo con interés sólo por el aspecto psicológico del individuo (James y Jones, 1974; Halpin y Croft, 1963) y, finalmente una tercera corriente la cual se preocupa de la percepción que el trabajador tiene tanto de la estructura como de los procesos que ocurren en su lugar de trabajo (Litwin y Stringer, 1968; Peiró, 1990; Mellado, 2005). Según esta última corriente, la forma de comportarse de un individuo en un trabajo no depende sólo de sus características personales sino también de la forma en que éste percibe su ambiente de trabajo y los componentes de su organización, por lo que el clima organizacional "constituye una configuración de las características de una empresa, así como las características personales de un individuo constituyen su personalidad" (Brunet, 1992). Podemos decir que el clima organizacional es también la forma de personalidad que caracteriza a una empresa.

Existen varios estudiosos del clima organizacional; destacamos a Rensis Likert (Chiavenato, 2002), conocido en el mundo por sus trabajos en psicología organizacional, elaboración de cuestionarios e investigaciones sobre los procesos administrativos. Es uno de los autores que nos ofrece una de las teorías más completas por su nivel de explicación y de extrapolación; su teoría nos permite visualizar en términos de causa y efecto la naturaleza de los climas que se estudian y permite también analizar el papel de las variables que conforman el clima que se observa. La teoría del clima organizacional de Rensis Likert sostiene que el comportamiento de los trabajadores en la organización es producto del comportamiento administrativo y las condiciones organizacionales que éstos perciben, así como por sus informaciones, sus percepciones, sus esperanzas, sus capacidades y sus valores; es decir, para los trabajadores lo que cuenta es la forma cómo ven las cosas y no la realidad objetiva (Likert, 1932).

Dentro de los diversos factores que influyen en la forma de percepción individual del clima están la posición jerárquica que se ocupa dentro de la organización, el salario que se obtiene, los factores personales (personalidad, actitudes y nivel de satisfacción), los parámetros ligados al contexto, a la tecnología y a la estructura misma del sistema organizacional. Para Likert existen tres tipos de variables que determinan las características de una organización y que condicionan su clima: variables causales, variables intermedias y variables finales.

Variables causales: Llamadas también "independientes", susceptibles de sufrir modificaciones por los responsables de la organización tales como: la estructura de la organización y su administración: reglas, decisiones, etc. Éstas se distinguen por dos rasgos esenciales: modificables por los miembros de la organización y son variables de causa y efecto, es decir, su modificación altera otras pero no sufren generalmente la influencia 
de las otras variables. Las variables intermedias designan los estados y procesos subjetivos que se intercalan entre los estímulos y las respuestas: las motivaciones, las actitudes, el rendimiento, la eficiencia de la comunicación y la toma de decisiones, etc. Las variables finales, conocidas también como "dependientes", ya que resultan del efecto precedente de las dos anteriores. Reflejan los resultados obtenidos por la organización, ejemplo de estas son la productividad, los gastos de la empresa, las ganancias y las pérdidas, las cuales constituyen la eficacia organizacional de una empresa.

Likert afirma que la combinación y la interacción de estas variables permiten determinar dos grandes tipos de clima organizacional, cada uno de ellos con dos subdivisiones. Los climas obtenidos se sitúan sobre un continuo que se desplazan desde un sistema muy autoritario a un sistema muy participativo.

\section{Clima de tipo autoritario}

Sistema I. Autoritarismo explotador: En éste las decisiones y los objetivos se toman y trazan en la cima de la organización, distribuyéndolas de manera puramente descendente; la comunicación no existe más que en forma de directrices y de instrucciones específicas. Los empleados trabajan en un ambiente de miedo, castigos, amenazas y, ocasionalmente de recompensas (Rodríguez, 1999), las pocas interacciones entre superiores y subordinados se establecen con base en el miedo y la desconfianza. A pesar de los procesos de control fuertemente centralizados, generalmente se desarrolla una organización informal que se opone a los fines de la organización formal.

Sistema II. Autoritario paternalista: La dirección muestra una confianza condescendiente con sus empleados, como la de un amo con su siervo. La mayoría de las decisiones se toman en la cima, algunas en los niveles inferiores. Las recompensas y los castigos son los métodos para motivar o sancionar a los trabajadores y la dirección juega con las necesidades sociales que tienen sus empleados, transmitiendo la impresión de trabajar dentro de un ambiente estable y estructurado. Puede desarrollarse una organización informal pero ésta no siempre reacciona a los fines formales de la organización.

\section{Clima de tipo participativo}

Sistema III. Consultivo: La dirección tiene confianza en sus empleados. Las decisiones se toman en la cima permitiendo decisiones específicas en niveles inferiores. La comunicación es de tipo descendente con moderada interacción superior - subordinado. Las recompensas y los castigos ocasionales se utilizan para motivar a los trabajadores, buscando satisfacer las necesidades de prestigio y estima. Se basa en procesos de control delegados descendentemente con un sentido de responsabilidad en los niveles superiores e inferiores; la administración trabaja en la forma de objetivos por alcanzar.

Sistema IV. Participación en grupo: Se caracteriza porque la dirección tiene plena confianza en sus empleados. La toma de decisiones está diseminada en todos los niveles de la organización y la comunicación es de tipo ascendente, descendente y de forma lateral. Los empleados son motivados por su participación e implicación al establecer objetivos de rendimiento, mejoramiento de los métodos de trabajo y por la evaluación del rendimiento en función de los objetivos. Los grupos formales e informales son generalmente los mismos ya que empleados y directivos trabajan en equipo para alcanzar los objetivos y metas de la organización.

La teoría de Likert adopta los calificativos abierto/cerrados con respecto a los climas que existen dentro de una organización. Un clima abierto corresponderá a una organización dinámica, capaz de alcanzar sus objetivos, que procura satisfacer las necesidades sociales de sus miembros y donde éstos interactúan con la dirección en la toma de decisiones. El clima cerrado corresponde a una organización burocrática y rígida cuyos empleados experimentan insatisfacciones ante su labor y frente a la empresa misma. Los sistemas I y II corresponden a un clima cerrado y los sistemas III y IV a un clima abierto. Existe también 
correspondencia entre la teoría $\mathrm{X}$ y $\mathrm{Y}$ de McGregor y la clasificación de los climas de Likert, así la teoría X caracteriza a los sistemas I y II, y la teoría Y a los sistemas III y IV.

El carácter multidimensional del clima organizacional hace que las variables que lo integran sean numerosas y difíciles de aislar, por eso al momento de analizarlo y evaluarlo han existido divergencias entre los teóricos en cuanto a las variables que deben incluirse. Likert, incluyó ocho aspectos para su estudio: métodos de mando, motivación a los empleados, tipos de comunicación, cumplimiento de objetivos, proceso de toma de decisiones $\mathrm{y}$ distribución de responsabilidades, procesos de planificación, procesos de control, objetivo de rendimiento, perfeccionamiento referidos a la planificación y formación deseada.

Por su parte Litwin y Stringer (1968), consideraron las variables: responsabilidad individual, la remuneración, el riesgo y toma de decisiones, apoyo y tolerancia al conflicto. Pritchard y Karasick (1973) utilizaron once dimensiones: autonomía, conflicto y cooperación, relaciones sociales, estructura, remuneración, rendimiento, motivación, status, centralización de la toma de decisiones y flexibilidad de innovación; mientras que Halpin y Crofts (1963) utilizaron ocho dimensiones en un estudio realizado en una escuela pública, de las cuales, cuatro apoyaban al cuerpo docente y cuatro al comportamiento del director; estas dimensiones fueron: desempeño, sentimiento del docente al realizar las tareas rutinarias, espíritu, actitud distante e; importancia de la producción, comportamientos autoritarios $\mathrm{y}$ centrados en la tarea del director, confianza y, consideración.

En España cabe destacar la aportación del profesor Gallo (1993), para quien inciden en ciertos aspectos diferenciales de la cultura de la empresa familiar, cuestiones como, el mayor número de interesados $\mathrm{y}$ de interrelaciones existentes entre ellos; la diferente ostentación que se hace del poder durante la primera y la segunda generación familiar ; el compromiso distinto que existe por parte de los accionistas para con la empresa y entre ellos; las conexiones entre las cambiantes necesidades de los miembros de la familia y los propios ciclos evolutivos de la empresa. Somos conscientes de la dificultad para evaluar y definir un clima organizativo en la empresa familiar. Esta complejidad proviene del gran número de variables explicativas existentes, de las relaciones entre ellas y del cambio que experimentan a través del tiempo. Con esta premisa y con el objetivo de analizar los diferentes climas organizativos presentes en la empresa familiar se realizó una encuesta presente en el anexo al trabajo.

\section{Metodología: el cuestionario, su consistencia y la unidad de análisis}

El cuestionario se elaboró para conocer los posicionamientos de los integrantes de la familia empresaria respecto de ocho pilares o constructos, cuyo análisis global pretendía medir la cultura organizativa tal y como definimos en las líneas anteriores. Nos encontramos ante una muestra dirigida, existe ausencia de aleatoriedad en su selección, por tratarse de empresas familiares que asisten a los cursos de formación impartidos por la Cátedra Prasa de Empresa Familiar de la Universidad de Córdoba. El análisis de la encuesta nos ofrece un posicionamiento de la empresa familiar sobre cultura organizativa, pudiendo utilizar el test como herramienta de diagnóstico de la empresa familiar.

Los ocho pilares o constructos analizados abarcan aspectos como nepotismo, transparencia informativa, planificación, adaptabilidad, armonía familiar, toma de decisiones participativas. Cada constructo está formado por una serie de pronunciamientos a los que los encuestados deben responder en una escala cualitativa (siempre, frecuentemente, a veces, rara vez, jamás). El óptimo lo encontramos en una elección del vocablo "siempre" sobre el pronunciamiento valorado, mientras que la situación peor es recogida por la elección del vocablo "jamás".

La consistencia del cuestionario y su coherencia en la medida de los objetivos planteados se evalúa mediante el parámetro alfa de cronbach. Los análisis ejecutados dieron un 
alto grado de coherencia, con resultados, todos ellos por encima del 0.8 .

La unidad de análisis es la empresa familiar y no así el miembro o individuo, por lo que la variable a estudiar es el promedio obtenido por empresa familiar en cada uno de los pilares especificados, evitando así que el número de integrantes en una empresa pudiera afectar al resultado y buscando la coherencia con el objetivo perseguido en esta investigación.

El primero de los constructos identificado como objetividad, analiza en que grado perciben los miembros de una empresa familiar la existencia de nepotismo en su organización. Sobre nepotismo son muchos los autores que han escrito. Existe coincidencia en conceptualizarlo como el favoritismo concedido a un familiar; decisión tomada en base a relaciones emocionales más que en competencias del individuo (Schneider, 2000). Este constructo está constituido por siete pronunciamientos, 1 de ellos planteados en reverso como control de las respuestas.

El segundo constructo analiza la transparencia, lo forman 8 pronunciamientos, tres de ellos planteados en reverso, como mecanismo de control de las respuestas de los ítems muestrales. No es difícil encontrar en la literatura sobre empresa familiar textos en los que la ausencia o falta de transparencia justifica en parte el fracaso del relevo generacional (Rodríguez Alcaide y Rodríguez Zapatero, 2008). En la teoría de las organizaciones podemos hallar dos posturas bien diferenciadas sobre el concepto de transparencia. Una posición llamada conductual donde la transparencia es innata al buen desempeño de la organización. Son organizaciones que reducen la incertidumbre y generan una discusión honesta entre las partes. Los partícipes conocen los valores organizacionales y tienen por tanto mayor confianza para actuar y tomar decisiones. La otra postura plantea que las organizaciones son espacios donde los seres humanos interactúan en muchos sentidos y esta relación social se construye a partir de una gran complejidad que va más allá del control y la supervisión; es decir, la gente se relaciona dentro de la organización a partir de normas pero también a partir de encuentros, afinidades, odios y amores, etc. Sin duda, la empresa familiar en el concepto de transparencia, encontrar mejor lugar en esta segunda postura.

El tercer constructo objeto de análisis, se centra en analizar en qué grado está presente la planificación en la familia empresaria. Formado por catorce pronunciamientos, siete de los cuales están planteados en reverso. Planificar es vital para la supervivencia empresarial. En el caso de la empresa familiar esta planificación abarca un campo mayor que en empresas no familiares; no podemos olvidar que existen cuestiones familiares que afectan al negocio, tales cómo criterios para la entrada al negocio de familiares, formación de familiares, planificación de la sucesión en gestión, propiedad etc. La relación positiva entre planificación y supervivencia es incuestionable.

El constructo cuarto, quinto y sexto correspondientes a los conceptos flexibilidad, orientación al cliente y aprendizaje respectivamente; son constructos netamente empresariales, que deben estar presentes en cualquier organización que busque la eficacia y la eficiencia de su sistema. La armonía familiar es el séptimo de los constructos bajo análisis; lo forman doce pronunciamientos, seis de ellos en orden reverso como control a las respuestas. De acuerdo a Belausteguigoitia (2003) la empresa familiar tiene su centro en el concepto de armonía cuya etimología proviene del griego harmós, que significa concordancia, ajuste. La Cátedra de Empresa Familiar IESE, Universidad de Navarra (2008) en su Newsletter 35 define el concepto de armonía como el estado de afecto y buena correspondencia que deberían compartir en todo momento los miembros de una familia empresaria para asegurar la buena marcha del negocio familiar. Para lograr la armonía familiar existen tres elementos fundamentales:

- La cohesión

- La adaptabilidad

- La comunicación en la familia

La cohesión familiar es el vínculo emocional que los miembros de la familia tienen entre sí y la adaptabilidad sería la habilidad de la familia para cambiar y adaptarse en la presencia de 
cambios importantes. Existen varios conceptos vinculados con la cohesión familiar como son el cariño entre familiares, la implicación familiar, la unión de padres e hijos y el respeto. De la misma manera, la adaptabilidad de la familia va de la mano de un buen manejo de la disciplina, un buen liderazgo, la capacidad de entendimiento entre los miembros de la familia, así como tener claros los roles y las reglas familiares. Del mismo modo, para que la cohesión y adaptabilidad puedan desarrollarse en una familia, la buena comunicación es indispensable. Una buena comunicación nace de la empatía entre los miembros de la familia, la escucha activa, la libertad de expresión, así como la consideración y el respeto entre los miembros de la familia.

El último de los constructos analizados es la participación en la toma de decisiones. Lo forman siete pronunciamientos, tres de ellos planteados en reverso. La forma o manera en que se toman y ejecutan las decisiones en una empresa está directamente relacionado con el resultado de la misma (Rodríguez Alcaide y Rodríguez Zapatero, 2006). Es por ello necesario e importante conocer de qué manera son tomadas las decisiones en la empresa familiar. El estilo de dirección forma parte de la cultura organizativa de toda empresa por lo que era un constructo a valorar.

\section{Resultados del estudio.}

El número de encuestados fue de 105 alumnos pertenecientes a 23 empresas familiares, 15 de ellas en fase de directorfundador $(65 \%)$ y las 8 restantes en sociedad de hermanos $(35 \%)$. La edad media de la muestra es de 39 años con una desviación típica de 14 años. En lo que a género se refiere, los hombres representan el $58 \%$ de la muestra mientras que el $42 \%$ restante son mujeres. Un $22.73 \%$ de las empresas de la muestra tienen presencia en mercados internacionales, el $40.91 \%$ de las empresas dirige sus productos al mercado nacional, el $31.82 \%$ actúa a nivel regional y tan sólo un $4.55 \%$ restringe su negocio a nivel local. En cuanto a tamaño, medido por el nivel de facturación anual ${ }^{1}$, la muestra presenta la siguiente estructura. Un $32 \%$ son microempresas, las empresas pequeñas representan el $54 \%$ mientras que el $9 \%$ y el $6 \%$ corresponden a medianas y grandes respectivamente.

Para obtener una primera aproximación visual del posicionamiento de las 23 empresas familiares bajo estudio, se realizó un gráfico diferencial semántico ${ }^{2}$. En el eje de abscisas aparece la valoración del constructo en un intervalo del cero al treinta ${ }^{3}$. En el eje de ordenadas los 8 constructos analizados. Los puntos representan la valoración media de cada empresa familiar para cada constructo bajo estudio, mientras que la línea, representa la media de las 23 empresas familiares. La primera conclusión que sacamos es la gran variabilidad de posicionamientos en todos los constructos e inclusive dentro de la misma empresa familiar los posicionamientos para cada constructo difieren sensiblemente en puntuación, pues la dispersión es notable.

Observando el gráfico 1 podemos destacar que los constructos de mayor variabilidad; es decir, donde mayor divergencia de posicionamientos encontramos, son la valoración de nepotismo y de profesionalidad. Esta situación nos da información sobre los dos constructos de la cultura organizativa que provocan mayor tensión y que deben ser

\footnotetext{
${ }^{1}$ Microempresas $<2$ millones de euros; pequeñas de 2 a 10 millones de euros, medianas entre 10 y 50 millones de euros, grades $>50$ millones de euros.

2 El psicólogo Charles E. Osgood desarrolló a finales de los años 50 un método de visualización denominado Semantic differential con el objetivo de representar gráficamente las diferentes connotaciones asociadas a un palabra en diferentes individuos. Osgood pidió a los participantes de sus estudios anotar una palabra en una serie de escalas que vaya de un extremo al otro (por ejemplo favorable/desfavorable). De la distancia observada entre los diferentes perfiles observados para individuos o grupos de individuos, Osgood dedujo la distancia psicológica y eventualmente conductista entre los individuos o grupos

${ }^{3}$ Para obtener la valoración numérica de cada constructo se procedió a cambiar la escala cualitativa por una cuantitativa con valores comprendidos entre 1 y 5 . Siendo 1 jamás y 5 siempre.
} 
Diferencial semántico de los constructos analizados

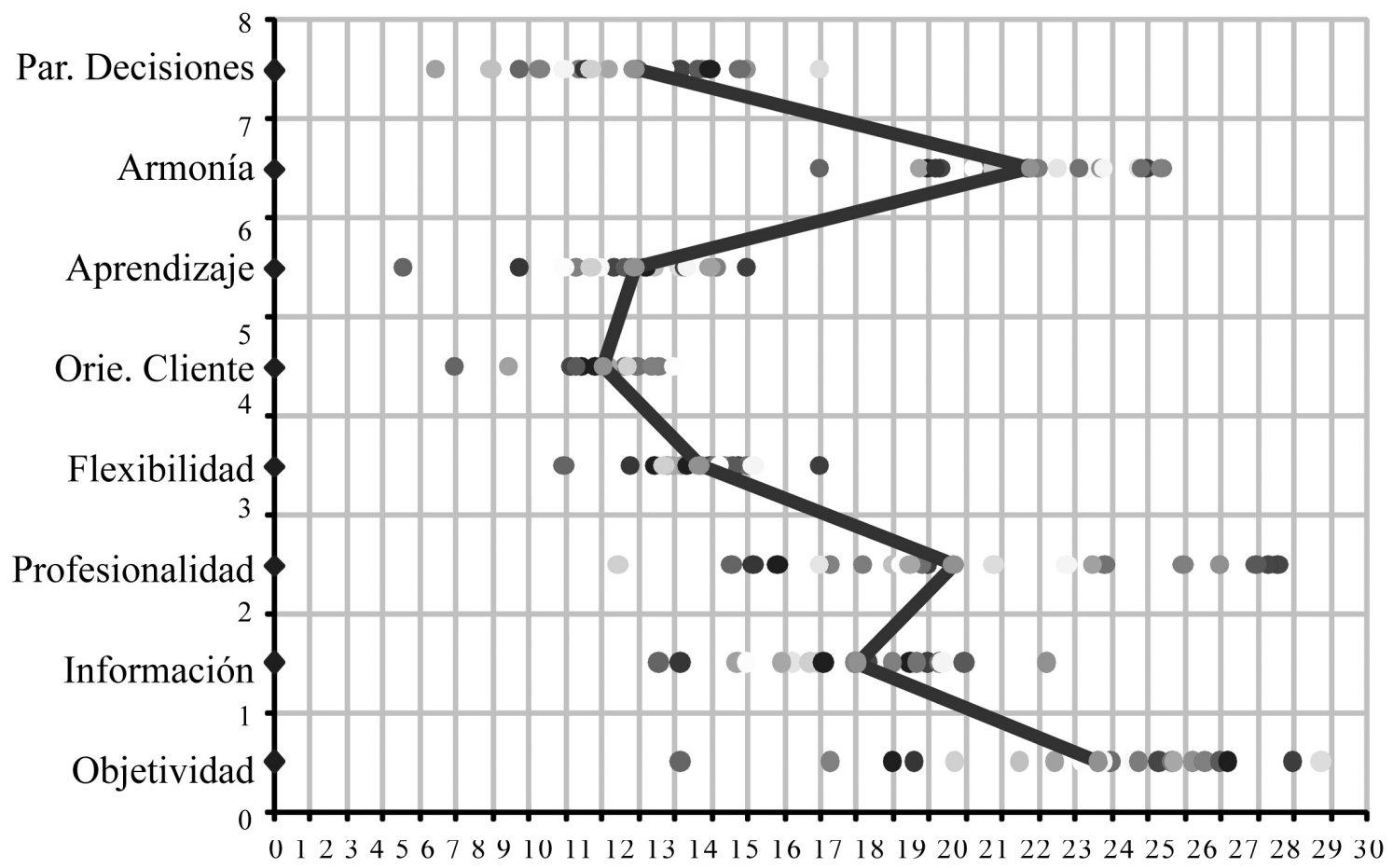

estudiados en aras de mejorar la supervivencia de la empresa familiar.

El segundo paso en nuestra investigación consistió en un análisis de la matriz de correlación para detectar posibles relaciones entre variables. Los resultados arrojaron una alta correlación entre las diferentes variables observadas, (véase tabla 1).

El valor del coeficiente de correlación varía entre -1 y 1 . Ambos extremos representan una correlación perfecta y 0 representa la ausencia de asociación. Cuanto más cercano al cero sea el coeficiente más débil será la asociación. Para analizar los coeficientes de correlación obtenidos haremos uso del baremo siguiente:

\pm 0.00 a 0.25 baja o ninguna correlación \pm 0.26 a 0.50 correlación moderada baja \pm 0.51 a 0.75 correlación modera alta \pm 0.76 a 1.00 alta o perfecta correlación

En base a los resultados obtenidos en el análisis de correlación, nuestro tercer paso en la investigación consistió en realizar un análisis de componentes principales con el objetivo de encontrar el posicionamiento de una empresa

\section{Tabla 1}

Coeficientes de correlación de los 8 constructos bajo estudio.

\begin{tabular}{|c|c|c|c|c|c|c|c|c|}
\hline & Objetividad & Información & $\begin{array}{l}\text { Profesiona- } \\
\text { lidad }\end{array}$ & Flexibilidad & $\begin{array}{l}\text { Orie. } \\
\text { Cliente }\end{array}$ & Aprendizaje & Armonía & $\begin{array}{l}\text { Part. } \\
\text { Decisiones }\end{array}$ \\
\hline Objetividad & 1,000 & 0,567 & $\mathbf{0 , 5 0 7}$ & 0,766 & 0,538 & 0,744 & $\mathbf{0 , 7 7 7}$ & 0,674 \\
\hline Información & $0,567^{(1)}$ & 1,000 & 0,574 & 0,644 & 0,418 & 0,545 & 0,656 & 0,529 \\
\hline $\begin{array}{l}\text { Profesiona- } \\
\text { lidad }\end{array}$ & $\mathbf{0 , 5 0 7}$ & 0,574 & 1,000 & 0,488 & 0,067 & $\mathbf{0 , 4 3 3}$ & 0,287 & 0,244 \\
\hline Flexibilidad & 0,766 & 0,644 & 0,488 & 1,000 & 0,494 & 0,776 & 0,734 & $\mathbf{0 , 5 0 1}$ \\
\hline $\begin{array}{l}\text { Orie. } \\
\text { Cliente }\end{array}$ & $\mathbf{0 , 5 3 8}$ & 0,418 & 0,067 & 0,494 & 1,000 & 0,405 & 0,627 & 0,501 \\
\hline Aprendizaje & 0,744 & 0,545 & $\mathbf{0 , 4 3 3}$ & 0,776 & 0,405 & 1,000 & 0,682 & 0,277 \\
\hline Armonía & $\mathbf{0 , 7 7 7}$ & 0,656 & 0,287 & 0,734 & 0,627 & 0,682 & 1,000 & $\mathbf{0 , 7 0 0}$ \\
\hline $\begin{array}{c}\text { Part. } \\
\text { Decisiones }\end{array}$ & 0,674 & 0,529 & 0,244 & $\mathbf{0 , 5 0 1}$ & $\mathbf{0 , 5 0 1}$ & 0,277 & 0,700 & 1,000 \\
\hline
\end{tabular}

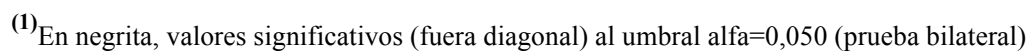


familiar en el mapa de la cultura organizacional, creando así una herramienta de diagnóstico y análisis de los problemas organizacionales de la familia empresaria.

La utilización de coordenadas factoriales permite tener un marco común en la formación de conglomerados o grupos de empresas familiares. El análisis de componentes principales consiste en expresar un conjunto de variables en un conjunto de combinaciones lineales de factores no correlacionados entre sí, dando cuenta estos factores, cada vez, de una fracción más débil de la variabilidad de los datos. Este método nos permite representar los datos originales (empresas familiares, constructos) en un espacio de dimensión inferior al original al mismo tiempo que limita la pérdida de información por dicha reducción. Es un análisis alternativo a la regresión que evita el uso de de variables redundantes y permite identificar la estructura de una población y los grupos o cluster que la forman.

La tabla 2 recoge los valores propios, que están vinculados al siguiente concepto: la calidad de la proyección cuando pasamos de $n$ dimensiones (siendo $\mathrm{N}$ el número de variables) a un número menor.

Tabla 2

Valores propios de los factores.

\begin{tabular}{llll}
\hline & Valor & $\%$ & $\%$ \\
Constructos & propio & varianza & acumulado \\
\hline F1 & 4,890 & 61,121 & 61,121 \\
F2 & 1,106 & 13,821 & 74,942 \\
F3 & 0,716 & 8,946 & 83,888 \\
F4 & 0,469 & 5,857 & 89,745 \\
F5 & 0,377 & 4,718 & 94,463 \\
F6 & 0,214 & 2,673 & 97,136 \\
F7 & 0,140 & 1,754 & 98,889 \\
F8 & 0,089 & 1,111 & 100,000 \\
\hline
\end{tabular}

En nuestro caso, observamos que el primer valor propio es 4.89 y representa el $61 \%$ de la variabilidad, lo que significa que si representáramos en un solo eje a nuestras empresas familiares tendríamos explicado el $61 \%$ de la variabilidad. A cada valor propio le corresponde un factor. Cada factor es en realidad una combinación lineal de las variables iniciales. Lo ideal es que los dos factores primeros representen un alto grado de la variabilidad de la muestra estudiada. En nuestro caso el $74.94 \%$. . En el gráfico 2 se muestra el biplot de correlación (plano vectorial de las variables). Representa una proyección de las variables iniciales sobre un plano de dos dimensiones constituido por los dos primeros factores.

Las flechas deben interpretarse como direcciones de crecimiento de las variables en el espacio factorial. Empresas familiares con orientación al cliente tienen una toma de participación activa. El centro del gráfico representa la media de todas las variables, a partir de este centro los vectores crecen en un sentido y decrecen en el sentido opuesto. Dos variables están relacionadas si el ángulo que forman es nulo $0^{\circ}$ (paralelas). Es el caso de las variables aprendizaje e información. Dos variables están relacionadas de manera negativa si su ángulo es de casi $180^{\circ}$ (opuestas) y dos variables no están asociadas si su ángulo es cercano a $90^{\circ}$ (perpendicular). Mientras más cercano al centro, (vector pequeño) la variable no es relevante $y$ si la eliminamos no acontecerían cambios sustanciales en el modelo.

Para verificar qué variables forman los factores es necesario analizar la tabla de cosenos; cuanto más elevado sea el coseno (en valor absoluto) más vinculada está la variable al factor.

El factor 1 (F1) tiene vinculadas objetividad, flexibilidad y armonía. El factor 1 se puede definir como un eje construido por la ley máxima para optimizar la toma de decisiones y su ejecución; es decir, un ambiente en que los componentes del equipo de dirección trabajan con objetividad, flexibilidad y en armonía familiar.

Podemos considerar que el factor 1 viene explicado por temas familiares que inciden en la cultura empresarial. Mientras que el factor 2 tan sólo representa mayor grado de vinculación a la variable profesionalidad. Recordemos que este constructo analiza el grado de planificación existente en la familia empresaria. 


\section{Gráfico 2}

Proyección de las variables estudiadas en el plano factorial.

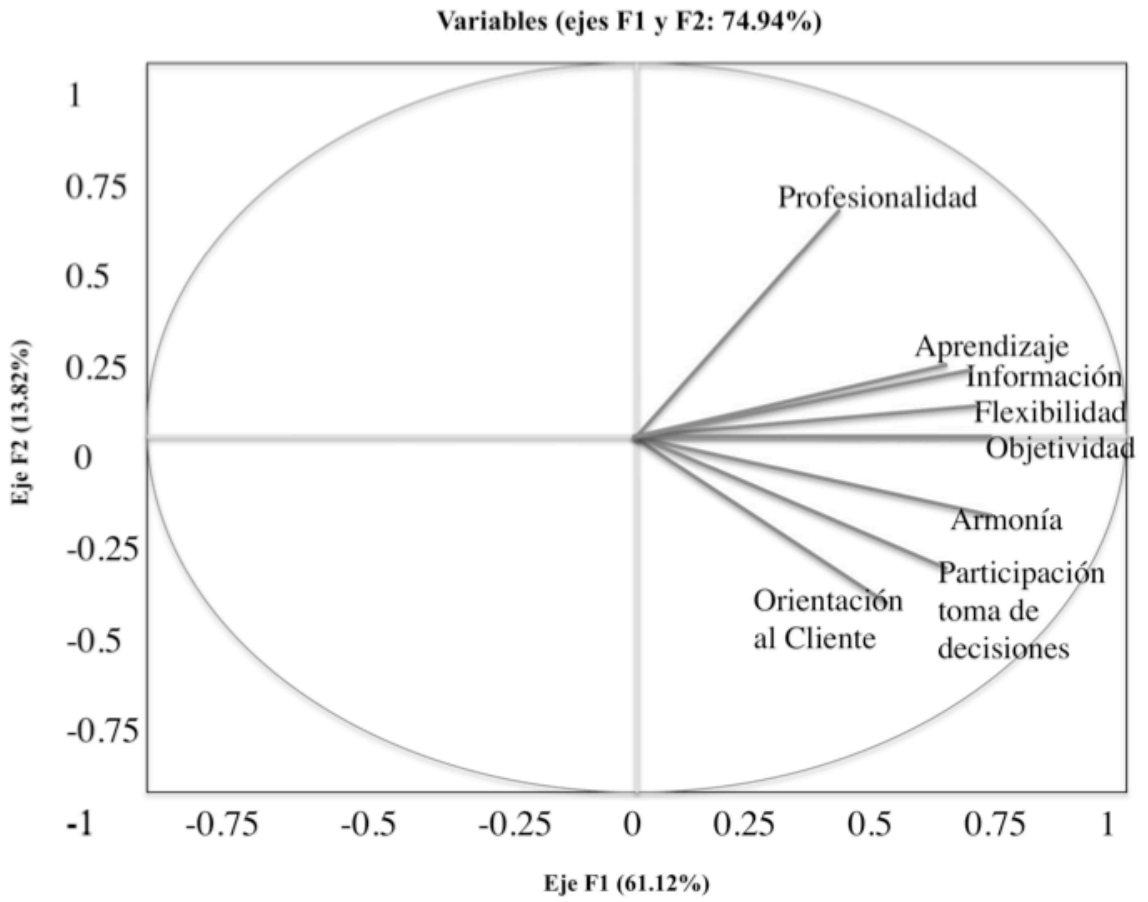

El gráfico 3 corresponde al biplot de distancias (Plano escalar de las observaciones, empresas familiares). Los puntos representan a las familias empresarias. Permite representar a las empresas familiares en un plano de dos dimensiones e identificar tendencias. Podemos observar qué empresas familiares están bien representadas en los ejes a partir de la tabla de cosenos cuadrados y las contribuciones.

\section{Gráfico 3}

Empresas familiares en la dimensión factorial.

Observaciones (ejes F1 y F2: 74.94\%)

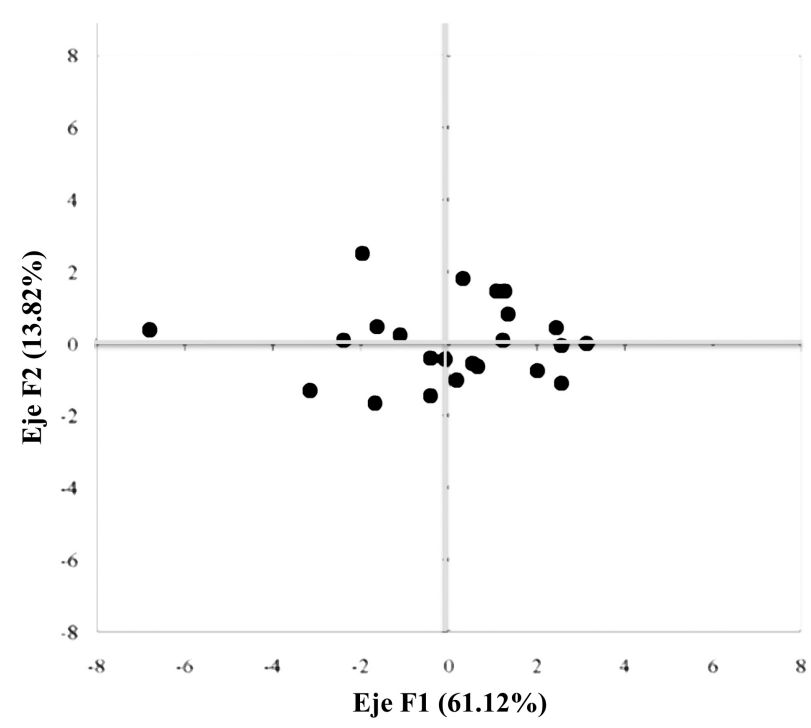

Las empresas familiares que se encuentran cercanas en el espacio de dos dimensiones, son empresas con similares posicionamientos respecto de los constructos estudiados. En un biplot simétrico representamos las variables y las empresas familiares de forma simultánea en el subespacio factorial de dimensión reducida (véase gráfico 4).

\section{Gráfico 4}

Empresas familiares y variables de la cultura en la plano factorial.

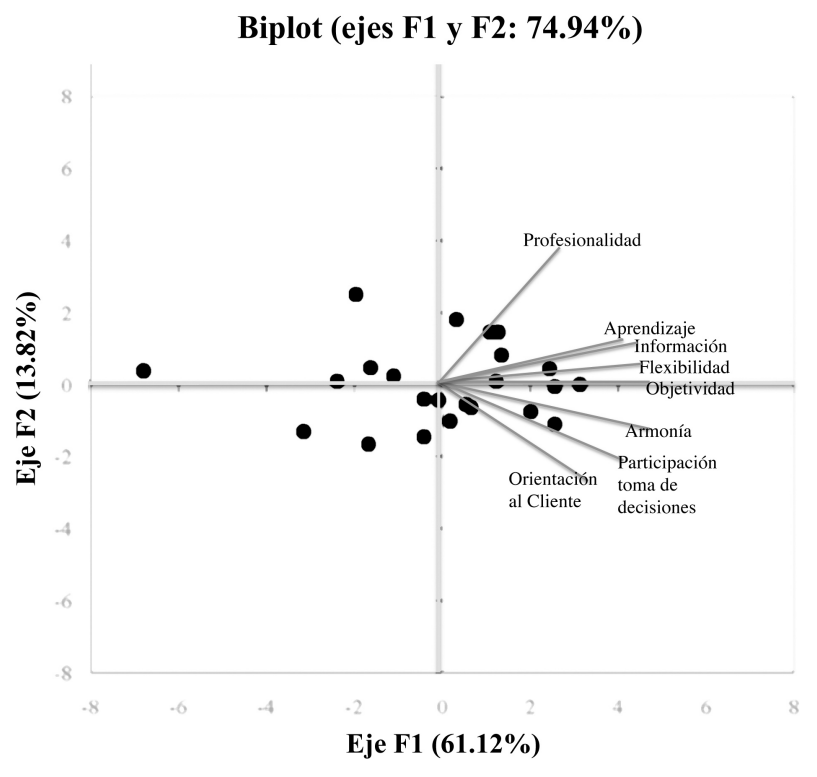

Rodríguez Zapatero, M. (2011). Mapa de la cultura organizativa de la empresa familiar: un estudio de casos en la provincia de Córdoba. Revista de Empresa Familiar, 1(2), 59-70. 
Este gráfico suele interpretarse diferenciando entre los cuatro cuadrantes. Las empresas familiares más alejadas del origen de coordenadas presentan el perfil más alejado del perfil medio de las empresas familiares estudiadas.

Según lo anterior podríamos identificar dos grupos de empresas familiares; las primeras situadas en los cuadrantes nordeste y sureste y las segundas en el noroeste y suroeste.

Con este análisis de componentes principales obtenemos dos grupos claros de empresa familiar (dimensión 1 y dimensión 2).

La importancia de este trabajo reside no tanto en la clasificación de la empresa familiar según su cultura organizativa sino en la posibilidad de detectar qué empresas deben mejorar, en qué deben mejorar, y cuál es la dirección de mejora.

Podemos destacar la empresa familiar situada más al oeste, la plusvariante, se trata de una empresa familiar en segunda generación para tercera donde los problemas familiares $\mathrm{y}$ empresariales son patentes, lo que explica su posicionamiento en el mapa factorial.

Cuando de cultura se trata, las variables tiempo y entorno han de ser consideradas. Según evoluciona el entorno en el tiempo así evoluciona la organización.

Bajo esta hipótesis de partida decidimos observar la cultura organizativa discriminando por etapa generacional, obteniendo en el análisis de componentes principales dos factores diferentes según estemos en primera generación o en sociedad de hermanos y una mayor capacidad de explicación de la variabilidad explicada por un solo factor a medida que las generaciones empresariales van sucediéndose (véase gráficos 5 y 6 ).

En el análisis de la cultura organizacional considerando sólo a empresas en etapa de director-fundador, los dos factores obtenidos explican el 67, 36\% de la variabilidad.

Por otro lado, en la sociedad de hermanos tan sólo el factor uno aglutina el $73 \%$ ascendiendo al $86 \%$ de variabilidad explicada cuando usamos los dos factores.

\section{Gráfico 5}

Empresas familiares de primera generación en el plano factorial.

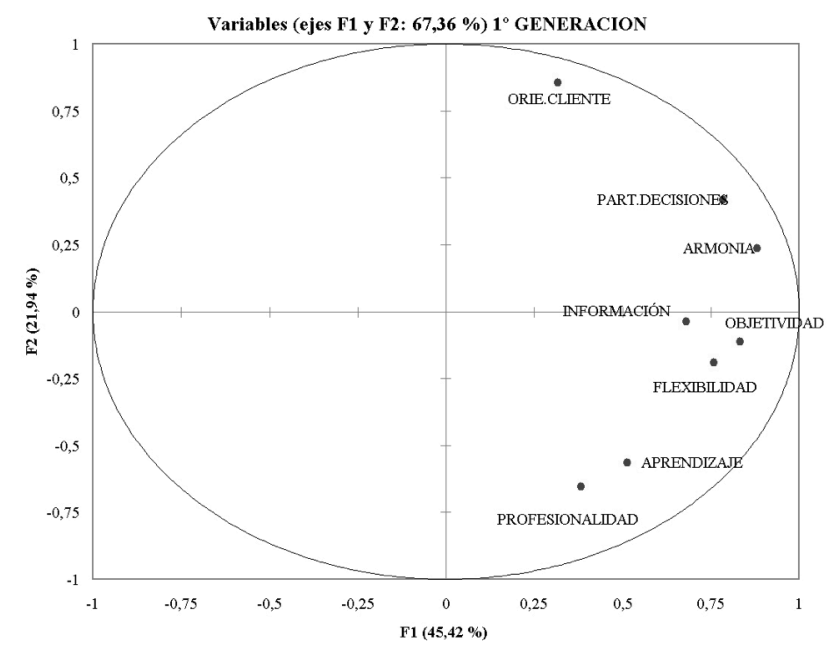

\section{Gráfico 6}

Empresas familiares de segunda generación en el plano factorial.

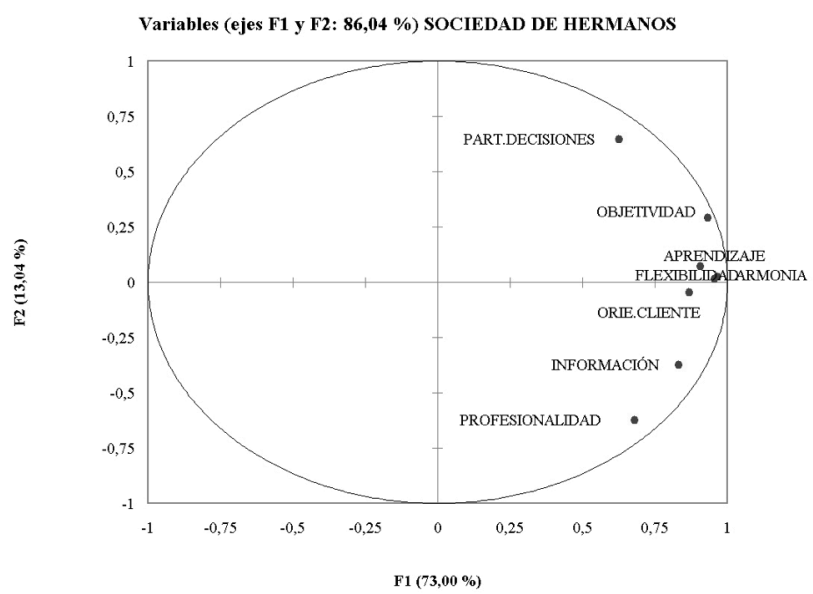

Así mismo, hay que destacar que las variables asociadas a cada factor varían según se trate de una u otra generación. En empresas familiares en primera generación el factor 1 está ligado a la armonía, objetividad, participación en decisiones con altos valores en la tabla de cosenos, mientras que el factor 2 sólo muestra relación con la variable orientación al cliente.

En el caso de empresas familiares en sociedad de hermanos, el primer factor tiene asociadas las variables armonía, flexibilidad, aprendizaje, orientación al cliente y objetividad mientras que el segundo factor se asocia con la participación en decisiones. Cabe destacar que tanto en primera como segunda generación, armonía y objetividad son básicas en la explicación de la cultura organizativa. Esta 
situación es lógica tal y cómo se ha definido la armonía y la objetividad. Estas dos variables deben estar siempre presentes en la cultura organizativa independientemente de la generación de que se trate.

Cuando analizamos la composición de variables del factor 2, el cambio entre generaciones es importante y determinante. En empresas familiares de primera generación la orientación al cliente es explicativa de la cultura. En el caso de empresas en sociedad de hermanos la orientación al cliente es sustituida por la participación en decisiones. Las hipótesis con las que se trabaja es que para pasar de primera generación a segundad generación la orientación al cliente es vital.

Si la empresa sobrevive la orientación al cliente es sustituida por una toma de decisiones participativa, algo que se justifica por el crecimiento en número de personas involucradas en la toma de decisiones. Ello no implica que la orientación al cliente desaparezca de la cultura organizativa sino que no es una variable decisiva en segunda generación ya que una empresa no sobrevivirá si no está orientada al cliente sea o no familiar.

\section{Conclusiones}

La cultura organizativa de la empresa familiar viene definida por un conjunto de variables interrelacionadas entre sí. Es obvio que un número elevado de variables genera mayor complejidad en el análisis. Las herramientas matemáticas utilizadas en este trabajo tratan de minimizar dicha complejidad.

La cultura organizativa de la empresa familiar cambia con el trascurso del tiempo. Los análisis de componentes principales realizados para empresas familiares en primera y segunda generación arrojan 2 factores explicativos de la cultura con composición diferente de variables. Luego no podemos considerar la cultura organizativa desde una perspectiva estática sino como algo que evoluciona y se desarrollo con el tiempo.

Los posicionamientos de cultura organizativa de las empresas familiares son variados no podemos hablar de un patrón repetitivo lo que viene a confirmar la hipótesis de partida que la cultura organizativa de una empresa familiar es única e inimitable, la empresa familiar es singular.

La armonía familiar influye de manera decisiva y progresiva en la cultura organizativa desarrollada en la familia empresaria durante el transcurso del tiempo. El papel de esta variable adquiere mayor importancia conforme se suceden las generaciones. Esta conclusión se deduce de la tabla de cosenos encontrada en primera y segunda generación. Así mismo hay que destacar la correlación perfecta y positiva que existen en empresas familiares entre flexibilidad y armonía. Podemos concluir que trabajar la flexibilidad en la familia empresaria posibilita la existencia de una armonía familiar.

A medida que evoluciona la familia empresaria las variables de su mapa de cultura organizativa afectan en mayor medida aglutinando el análisis factorial mayor explicación de la variabilidad a medida que se suceden generaciones.

La principal limitación al presente estudio proviene de un tamaño muestral que no permite realizar inferencias a la población y el tratarse de una muestra dirigida. No obstante, los resultados obtenidos permiten concluir que la cultura organizativa de toda familia empresaria viene determinada por una amalgama de variables que inyectan una gran complejidad en el análisis. La cultura organizativa de la empresa familiar evoluciona con el transcurso del tiempo y los cambios que acontecen, luego un análisis eficiente de la cultura en la familia empresaria no puede limitarse a un único momento en el tiempo, sino que por el contrario debe repetirse a medida que éste pasa y acontecen cambios en la empresa familiar.

\section{Bibliografía}

Belausteguigoitia, I. (2003). Empresas Familiares, su dinámica, equilibrio y consolidación. McGraw Hill.

Brunet, L. (1992). El clima en las organizaciones: definición, diagnóstico y 
consecuencias. Colección Desarrollo de Recursos Humanos. México: Editorial Trillas.

Cátedra de Empresa Familiar IESE, Universidad de Navarra (2008). Newsletter 35. IESE Business School, Universidad de Navarra.

Chiavenato, I. (2002). Gestión del talento humano. Colombia: McGraw - Hill.

Gallo, M. (1993). Cultura en empresa familiar. En: M. Gallo (Ed.): La empresa familiar 4, (225-249). IESE, publicaciones de la Cátedra de empresa familiar, Barcelona.

Gellerman, S.W. (1960). People, problems and profits. Nueva York. McGraw Hill.

Glick, W. H. (1985). Conceptualizing and measuring organizational and psychological climate: Pitfalls in multinivel research. Academy of Management Review,10 (3), 601-616.

Halpin, A. y Croft, D. (1963). The organizational climate of schools. Chicago: University of Chicago Press.

James, L.R. y Jones, A.P. (1974). Organizational climate: A review of theory research. Psychological Bulletin, 81, 1096-112.

Likert, R. (1932). A technicque for the measurement of attitudes. Archives of Psychology, 32 (144), 1-55.

Litwin, G. y Stringer, R. (1968). Motivation and organizational climate. Boston: Harvard Business School.
Mellado Ruiz, C (2005) El proceso comunicativo al interior de la pequeña empresa industrial: clima, liderazgo y realidad organizacional. Ediciones Universidad Pontificia de Salamanca: España.

Peiró, J. M. (1990). Organizaciones, nuevas perspectivas psicosociológicas. Barcelona: PPU.

Pritchard, R.P. y Karasick, B.W. (1973). The effects of organizational climates on managerial job performance and job satisfaction. Organizational behavior and human performance, 9, 126-146.

Rodríguez Alcaide, J.J y Rodríguez Zapatero, M. (2006). La empresa familiar: implicación y logro de sus constituyentes. Cátedra de Empresa Familiar. Universidad de Córdoba.

Rodríguez Alcaide, J.J y Rodríguez Zapatero, M. (2008). Confianza, comunicación y pacto en la familia empresaria. Cátedra de Empresa Familiar. Universidad de Córdoba.

Schneider, B. (2000). The psychological life of organizations. Ashkanas y N.M.

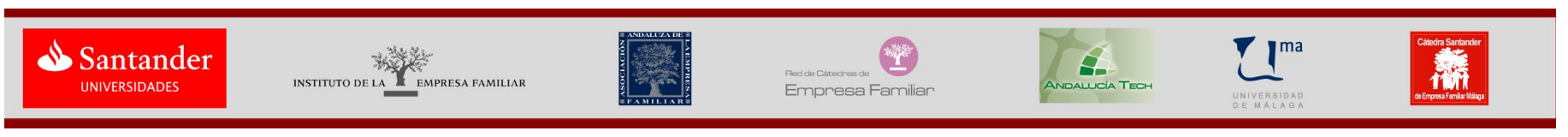

